\title{
Newly lncRNAs SNPs been Identified, What Exact Role will it Act in Cancer?
}

\section{Zhenjian Zhuo and Jing $\mathrm{He}^{*}$}

Department of Pediatric Surgery, Guangzhou Institute of Pediatrics, Guangdong Provincial Key Laboratory of Research in Structural Birth Defect Disease, Guangzhou Women and Children's Medical Center, Guangzhou Medical University, Guangzhou, Guangdong, People's Republic of China

*Corresponding Author: Jing He, Department of Pediatric Surgery, Guangzhou Institute of Pediatrics, Guangdong Provincial Key Laboratory of Research in Structural Birth Defect Disease, Guangzhou Women and Children's Medical Center, Guangzhou Medical University, Guangzhou, Guangdong, People’s Republic of China.
Received: May 26, 2020

Published: July 17, 2020

(C) All rights are reserved by Zhenjian Zhuo and Jing He.
More than $80 \%$ of the human genome is actively transcribed but only about $2 \%-3 \%$ is translated into proteins, indicating that the transcriptional output of human genome is almost monopolized by non-coding RNAs (ncRNAs) [1]. Cancer relevant ncRNAs are gradually becoming one of the hottest issues in the RNA biology and oncology [2]. Reports on ncRNAs are colorful and diversified. Many researchers have engrossed in the subject about ncRNAs including long noncoding RNAs (lncRNAs), microRNAs, and piRNAs, exploring their biological mechanisms and regulatory pathways in some complex diseases [3].

LncRNAs are generally defined as non-coding RNA molecules longer than 200 nucleotides. By now, more than 20,000 lncRNAs have been identified [4]. However, these studies are only the tip of the iceberg. LncRNAs are implicated as important regulators in numerous physiological and pathological processes [5]. But how does it play in cancer? Functionally, lncRNAs are classified into four categories based on their molecular mechanism of action, including signaling, decoy, guide, and scaffold lncRNAs [6]. Signal IncRNAs silence or activate other transcripts. Their expression is cell type-specific and under tight transcriptional control to respond to diverse stimuli. Therefore, they act as markers of functionally significant biological events. Decoy lncRNAs compete with transcription factors and RNA-binding proteins, or miRNA, for their interactions with specific targets (mostly act as a competitive endogenous RNA to compete with miRNA binding through complementary base pairing and reduce combined with miRNA to other target genes by sponging adsorption of endogenous miRNA). Guide IncRNAs recruit selected chromatin-modifying enzymes to their specific target sequence, either in cis or in trans. Scaffold IncRNAs bind multiple proteins together to keep them closer to ribonucleoprotein complexes, and stabilize nuclear structures or signaling complexes, or facilitate their action on histones. However, the above characterized functions of IncRNAs could not fully explain their role in cancer etiology.

SNPs are the most common type of genetic variation, nearly occupy about an incidence of 1 per 100-300 nucleotides. With the advancement of research, a large number of SNPs have been discovered. More than 10 million SNPs have been documented in the human genome [7]. Genome-wide association studies (GWASs) have identified more than 6500 disease-predisposing SNPs in the last decade [8]. Among them, only 7\% SNPs are located in proteincoding regions [9]. SNPs hold great potential as markers for prognosis, clinical outcome, drug resistance, and susceptibility of cancer. SNPs could change or disrupt the RNA secondary structure of the lncRNAs, affecting their molecular function and influencing their expression pattern.

Several mutated IncRNAs in cancers have been frequently detected, including HOTTIP, MALAT1, and HOTAIR. Moreover, SNPs located in these IncRNAs have been well documented, indicating the involvement of these SNPs in relevant cancer functions [10]. Bhartiya., et al. [11] detected that the distributions of the SNPs in lncRNAs present distinct patterns in potential functional elements. A SNP in a regulatory element might alter the abundance of a gene transcript. However, a plenty of SNPs are positioned in intronic or intergenic regions, and the relationship of these with lncRNA function in the cell context might be much more circumspect. To determine the function of SNPs in this respect, expression quantitative trait locus (eQTL) mapping can be adopted. With eQTL the relation of genotype data with RNA expression is measured to explore if a SNP is associated with gene transcription [12].

Our group mainly focus on the role of genetic variants on cancer risk, especially the lncRNAs SNPs. We have found that SNPs in IncRNAs H19 [13], Inc-LAMC2-1:1 [14], LINC00673 [15], and HOTAIR [16] could impact neuroblastoma risk. However, our study has some limitations, including a relative sample size, using a single population, not assessing the environmental effects, and not performing functional experiments. Actually, the above limitations are the common seen issues in most of the epidemiology study regarding lncRNAs SNPs and cancer risk. Solutions include: to expand the sample size (by lengthen the enrollment period or cooperating with other centers), to involve mix ethnicities, 
to incorporate the environmental effects, and to perform the biological mechanisms how the significant SNPs regulate cancer risk.

Therefore, the investigation of IncRNAs SNPs on cancer risk is still at its infancy stage. The substantial knowledge gap that still exists between the IncRNAs SNPs association and the molecular mechanism contributing to cancer risk represents both a question and an opportunity. In other words, this work is important for seeking the underlying genetic driver for cancer. The existed findings may inspire the scientists who are also interested in the genetic factors of cancer to validate the association of interest using more samples and potential biological mechanisms.

Due to the evolution of technology, it is likely that a host of discoveries on the involvement of lncRNAs SNPs in cancer are finally on the horizon. In the near future, clinical applications of lncRNAs SNPs might include early cancer diagnosis, risk prediction for relapse or progression, monitoring the effects of systemic therapies, and patient stratification. Eventually, these studies could also lead to the identification of novel targets for the development of innovative therapies. The research field for the study of IncRNAs SNPs involved in cancer is an unprecedented opportunity and challenge.

\section{Bibliography}

1. Anastasiadou E., et al. "Non-coding RNA networks in cancer". Nature Reviews Cancer 18.1 (2018): 5-18.

2. Fabbri M., et al. "Decrypting noncoding RNA interactions, structures, and functional networks". Genome Research 29.9 (2019): 1377-1388.

3. Gournay M., et al. "Regulat-INGs in tumors and diseases: Focus on ncRNAs". Cancer Letters 447 (2019): 66-74.

4. Consortium EP. "An integrated encyclopedia of DNA elements in the human genome". Nature 489.7414 (2012): 57-74.

5. Bhan A., et al. "Long Noncoding RNA and Cancer: A New Paradigm". Cancer Research 77.15 (2017): 3965-3981.

6. Kopp F., et al. "Functional Classification and Experimental Dissection of Long Noncoding RNAs". Cell 172.3 (2018): 393-407.

7. International HapMap C. "A haplotype map of the human genome". Nature 437.7063 (2005): 1299-1320.

8. Tam V., et al. "Benefits and limitations of genome-wide association studies". Nature Reviews Genetics 20.8 (2019): 467-484.

9. Ning S., et al. "Genome-Wide Mapping of SNPs in Non-coding RNAs". Advances in Experimental Medicine and Biology 1094 (2018): 39-48.
10. Gao P., et al. "Genomic Insight into the Role of lncRNA in Cancer Susceptibility". International Journal of Molecular Sciences 18.6 (2017).

11. Bhartiya D., et al. "Distinct patterns of genetic variations in potential functional elements in long noncoding RNAs". Human Mutation 35.2 (2014): 192-201.

12. Gupta RM., et al. "Mapping Novel Pathways in Cardiovascular Disease Using eQTL Data: The Past, Present, and Future of Gene Expression Analysis". Frontiers in Genetics 3 (2012): 232.

13. $\mathrm{Hu} \mathrm{C}$., et al. "Associations between $\mathrm{H} 19$ polymorphisms and neuroblastoma risk in Chinese children". Bioscience Reports 39.4 (2019).

14. Yang T., et al. "The rs $2147578 \mathrm{C}>\mathrm{G}$ polymorphism in the IncLAMC2-1:1 gene is associated with increased neuroblastoma risk in the Henan children". BMC Cancer 18.1 (2018): 948.

15. Li Y., et al. "Additional data support the role of LINC00673 rs11655237 C>T in the development of neuroblastoma". Aging (Albany NY) 11.8 (2019): 2369-2377.

16. Yang X., et al. "HOTAIR gene polymorphisms contribute to increased neuroblastoma susceptibility in Chinese children". Cancer 124.12 (2018): 2599-2606.

\section{Assets from publication with us}

- Prompt Acknowledgement after receiving the article

- Thorough Double blinded peer review

- Rapid Publication

- Issue of Publication Certificate

- High visibility of your Published work

Website: https://www.actascientific.com/

Submit Article: https://www.actascientific.com/submission.php Email us: editor@actascientific.com

Contact us: +919182824667 